\title{
Development Education - Globales Lernen - Global Citizenship Education. Bleibende Aporien und strategische Offerten im Weltkollektiv
}

\begin{abstract}
Zusammenfassung
Die aktuellen Transformationsherausforderungen der Welt werden zwischen weltgesellschaftlich-kommunikativen Offerten und weltgemeinschaftlich-interaktiven Optionen aufgespannt. Die damit verbundene Einheit der Differenz wird mit dem Konstrukt des Weltkollektives markiert. Dies ist der Ausgangspunkt für Fragen nach dem durch Bildung und Lernen erhoff- und erwartbaren Zukunftspotenzial, das Konzeptionen global orientierter Bildungsarbeit immanent ist. Damit wird der Rahmen in den Blick genommen, in dem sich Development Education, Globales Lernen und Global Citizenship Education entfalten können.
\end{abstract}

Schlüsselworte: Transformation, Weltgesellschaft, Weltkollektiv, Globales Lernen, Global Citizenship Education, Entwicklungspädagogik

\section{Abstract}

The current transformation challenges of the world span between communication offers of the world society and actionrelated options of the world community. The inseparable unity of their difference is marked by the construct of the global collective. This serves as the point of departure for questions concerning the future potential that is intended and expectable through education and learning and that is immanent to conceptions of globally oriented educational work. Thus, the type of framework moves into focus in which the concepts of development education, global learning and global citizenship education can flourish.

Keywords: Transformation, World Society, World Community, Global Collective, Global Learning, Global Citizenship Education, Development Pedagogy

\section{Einleitende Überlegungen}

Development Education, Globales Lernen und Global Citizenship Education (GCED) sind Konzeptionen, durch die mit verschiedenen Schwerpunktsetzungen auf Herausforderungen einer sich globalisierenden Weltgesellschaft reagiert werden soll (Scheunpflug \& Schröck, 2000; Lang-Wojtasik \& Klemm,
2017; Grobbauer, 2017; Treml, 2017; Surian, 2018; Bourn, 2020). Bereits in den kombinierten Begrifflichkeiten stecken eine Menge Fragen: Welche Verständnisse sind Bildung, Lernen, Pädagogik, Entwicklung, Bürger/-innentum und Globalität unterlegt und wie hängt alles zusammen? In welchen Traditionen und interdisziplinären Bezügen sind sie systematisch für Mensch und Welt verortet?

Das thematische Feld bleibt dynamisch und konzeptionelle Suchbewegungen zu Interkultureller, Friedens-, Menschenrechts- oder Umweltpädagogik sind ein bleibendes Debattenfeld (LfpB, 2019). Insbesondere Bildung für nachhaltige Entwicklung (BNE) hat mit der Verabschiedung der SDGs (Sustainable Development Goals) (UN, 2015) einen neuen Aufschwung bekommen. Bisher parallellaufende Debatten von EFA (Education for All) (UNESCO; 2020) und BNE haben im vierten Zielbereich intensive Berührungspunkte. GCED bietet mit seinen Wurzeln in der weltbürgerlichen Erziehung (Treml, 2011) und systematischen Bezügen bis hin zu politischer Bildungsarbeit (Wintersteiner et al., 2015) nachhaltige Anknüpfungspunkte an zukunftsfähige BNE (Lang-Wojtasik, 2020a).

Immer schnellere und unübersichtlichere Transformationsprozesse der Welt (WBGU, 2011) unterstreichen die Notwendigkeit, das Verhältnis gesellschaftlicher Herausforderungen und bildungsbezogener Optionen auf den Prüfstand zu heben (Clemens, Hornberg \& Rieckmann, 2019; Lang-Wojtasik, 2019a). Spätestens seit der Deklaration von Maastricht haben sich auf europäischer Ebene (O'Laughlin \& Wegimont, 2003) und mit dem Orientierungsrahmen Globale Entwicklung (KMK, BMZ \& Engagement Global, 2016) auch die nationalen politischen Rahmenbedingungen geändert. International ist der Versuch hervorzuheben, den Zusammenhang von Bildung für nachhaltige Entwicklung und Global Citizenship Education darzustellen (Bourn, Hunt \& Bamber, 2017; UNESCO, 2018). Auf europäischer Ebene werden strategische und Policy-bezogene Prozesse insbesondere vom Global Education Network (GENE) vorangebracht (Forghani-Arani, Hartmeyer, O'Loughlin \& Wegimont, 2013; Hartmeyer \& Wegimont, 2016; McAuley, 2018; Trindade Dolejšivá, 2018). Auf der Ebene deutscher Bundesländer gibt es umfassende Mög- 
lichkeiten, konzeptionelle Anliegen des Globalen Lernens und der GCED auch über die wachsende Implementierung der BNE voranzubringen (Overwien, 2016), die ein stetiges Ausbaupotenzial auf allen bildungsrelevanten Ebenen hat (Arnold, Carnap \& Bormann, 2017; Brock, de Haan, Etzkorn \& Singer-Brodowski, 2018; Müller-Christ, Giesenbauer \& Tegeler, 2018; BMBF, 2020).

Bei alledem bleiben ungelöste und möglicherweise unlösbare Herausforderungen erhoffter Transformation, die angesichts der Klimakatastrophe und Covid-19-Pandemie erneut zu Tage treten und den Gesamtdiskurs in verschiedener Intensität begleiten werden. In diesem Aufsatz geht es um die Re-Formulierung dieser Aporien und es werden strategische Offerten für die Zukunft angedeutet: (1) Welche Herausforderungen stellen sich in der aktuellen Transformationssituation? (2) Welche Optionen hat die Weltgemeinschaft, angesichts weltgesellschaftlicher Herausforderungen, ins Handeln für ein transformatives Weltkollektiv zu kommen? (3) Welcher zukunftsfähige Beitrag ist durch Bildung und Lernen für das Weltkollektiv im Sinne der UN-Transformationsagenda denkbar? (4) Welche konzeptionellen Offerten von Lernen erscheinen tragfähig, um das Bewusstsein von Menschen nachhaltig zu verändern, damit diese das Zusammenleben mit anderen und dem Planeten zukunftsfähig gestalten können? Diesen Fragen widmen wir uns in vier Thesen.

\section{These 1: Sichere Unsicherheit als Rahmen sozialer Ordnung}

Fundamentale Veränderungen auf dem Globus stellen die Lebensgrundlagen aller Menschen infrage und tragen zu Verunsicherungen bei.

Kaum etwas scheint derzeit sicherer zu sein als die zunehmende Wahrnehmung einer von Unsicherheit und Verunsicherung geprägten Welt, in der wir leben. Eigentlich war die angenommene „Normalität“ sozialer Ordnung nie anders. Möglicherweise haben wir es schlicht geschafft, den Herausforderungen stets fragiler Sicherheit einer sich immer stärker ausdifferenzierenden Gesellschaft semantisch zu begegnen und so die Unsicherheit als Grundlage verwundbarer Zivilisation aushalten zu können (Lang-Wojtasik, 2020d/i. E.).

Bei alledem hatten nationale Begrenzungen eine entscheidende Bedeutung, um internationale Grenzüberschreitungen als Erweiterung des eigenen Horizonts kennenzulernen und auch mögliche Handlungserfahrungen jenseits bekannten Terrains zu erproben. Sicherheit blieb dabei als Normalitätskonstrukt stets fragil und unsicher. Ein interessierter Blick hinter die Kulissen der großen Diskrepanzen zwischen globalem Norden und Süden sowie innerhalb von Weltregionen und Ländern eröffnete stets die Sicht auf schreiende Ungerechtigkeiten, die häufig intersektional verschränkt waren und sind. Globale Ungleichgewichte haben sich stets wiedergespiegelt: auf der einen Seite in der Verteilung von Ressourcen und Macht und auf der anderen Seite im Intensitätsgrad ihrer Auswirkungen auf verschiedene Teile der Welt. Dahinter liegen Fragen von Privilegien und Teilhabe (Datta, 2013). Gerade die Beschäftigung mit Fragen der De-Kolonisierung jenseits imperialistischer Weltgeschichte und -gestaltung nach 1945 oder entwicklungspolitische Bemühungen und der Ausbau internationaler Organisationen gaben der Idee einer Weltgemeinschaft neuen Auftrieb. Während die Menschheit heute insgesamt wohlhabender als je zuvor ist, ist die Welt weit von Gerechtigkeit oder Gleichwürdigkeit im Sinne der Menschenrechte entfernt und charakterisiert sich durch enorme Ungleichgewichte auf unterschiedlichen Ebenen. Der weltweit zunehmende Wohlstand ist der Motor global ansteigender Umweltzerstörung, wobei die reichsten 10\% der globalen Bevölkerung für mehr als die Hälfte der globalen CO2-Emissionen verantwortlich sind (Gore, 2020). Insbesondere unterliegt der enge $\mathrm{Zu}$ sammenhang zwischen Einkommen und Kohlendioxidausstoß gewaltigen Ungleichgewichten existierender Lebensstile und des Konsumverhaltens. Dabei sind diejenigen von den Klimawandelauswirkungen am meisten betroffen, die am wenigsten Kohlendioxid ausstoßen (Gore, 2015).

Der vielschichtig-interdisziplinäre Globalisierungsbegriff (Niederberger \& Schink, 2011) ist hilfreich, um die räumlichen Charakteristika der Welt, in der wir leben, für sachliche, zeitliche und soziale Facetten in den Blick nehmen zu können. Die Globalisierung hat ein hohes Maß an komplexer Vernetzung und wechselseitiger Abhängigkeit erreicht und lässt neben Chancen und Möglichkeiten vermehrt weltweite Herausforderungen kommunikativ in den Vordergrund treten. Globale Ungleichheit in ihrer sozialen und ökonomischen Dimension bleibt neben der globalen Erderwärmung eine der zentralsten Problematiken, denen endlich transformativ begegnet werden müsste. In dieser Aussage steckt eine implizite Handlungsaufforderung an Menschen, die gesellschaftlich expliziert und in politische, juristische, ökonomische usw. Agenden gegossen werden sollte.

Die vor fast fünfzig Jahren empirisch beschriebenen „Grenzen des Wachstums“ (Meadows et al., 1972) werden im fortschreitenden 21. Jahrhundert immer greifbarer (IPCC, 2018). Dahinter steht die Frage, ob sich die moderne Gesellschaft auf ökologische Gefährdungen einstellen kann (Luhmann, 1986), die jenseits nationalstaatlicher Grenzziehungen relevant sind. Damit zusammenhängend müsste geklärt werden, über welche Steuerungsmechanismen die moderne Gesellschaft verfügt und in welchem Verhältnis diese zu individuellen Entscheidungen für Veränderungen stehen. Immerhin gilt es, die seit langem beschriebene Klimakatastrophe mit all ihren Vernetzungen und Interdependenzen abzuwenden.

\section{These 2: Weltkollektiv zwischen Kommunikationsofferten und Handlungsoptionen}

Um Optionen großer Transformation im Sinne der SDGs zu erschließen, braucht es systematische Überlegungen zur Reichweite von Kommunikationsofferten (Weltgesellschaft) und Handlungsoptionen (Weltgemeinschaft) im Weltkollektiv.

Mit dem schwerfällig anmutenden Begriff des Weltkollektivs (Lang-Wojtasik, 2020b/i. E.) wird an dieser Stelle der spannungsreiche Zusammenhang von Gesellschaft und Gemeinschaft (im Anschluss an Tönnies, 1887/1922) auf die Ebene einer Weltbetrachtung gehoben, um soziale Ordnung als Grundlage und Rahmen globaler Herausforderungen begrei- 
fen zu können. In den Blick kommt die Einheit der Differenz systemtheoretisch beschriebener Weltgesellschaft (Kommunikationsofferten im Anschluss an Luhmann, 1971) sowie einer anthropologisch relevanten Weltgemeinschaft (Interaktionsoptionen im Anschluss an Plessner, 1924/2003). Das „Global Village" müsste sich demnach spannungsreich als ein abstraktes Kommunikationsangebot beschreiben lassen, zu dem konkrete Erprobungsoptionen in Gemeinschaft mit anderen in Beziehung gesetzt werden können (Lang-Wojtasik, 2014).

Die UN-Nachhaltigkeitsziele richten sich explizit an die Weltgemeinschaft und sind in ihrer radikalen Semantik für die Weltgesellschaft bisher beispiellos. Ob sie das Zeug haben, zu einer Charta („völkerrechtliches Grundgesetz“) für das Weltkollektiv der Menschen und des Planeten im 21. Jahrhundert zu werden (UN, 2015, S. 13), wird sich im weiteren Verlauf bis 2030 zeigen. Die ersten fünf Jahre nach ihrer Verabschiedung sind eher ernüchternd. Die fünf normativen Ps (People, Planet, Prosperity, Peace, Partnership) der Präambel, 17 Zielbereiche und 169 Teilziele sind das Ergebnis jahrelanger Beratungen, die zunächst kommunikativ relevant sind. Daher bleibt die Frage an die Weltgemeinschaft: Wie kommt es wo von wem zu Aktionen und Interaktionen, damit sich etwas verändert? Dieses würde ein umfassendes Ausmaß an Gemeinsinn erfordern, der Verzicht und Umkehr v. a. im globalen Norden bedeutete. Lassen sich damit Menschen zu Handlungen bewegen?

Deutlich wird, dass das Risiko und Variationsvielfalt mit der Entwicklung hin zu einer Weltgesellschaft als grenzüberschreitendem Kommunikationsangebot (Luhmann, 1971) stärker in den Mittelpunkt des Interesses gerückt werden. Kommunikative Offenheit wird zum räumlich eingrenzenden Rahmen sozialer Ordnung jenseits nationalstaatlicher Semantik, in dem alles denk- und vorstellbar ist. Damit einhergehen zunehmend wahrnehmbare Schwierigkeiten der Legitimation sachlicher Entscheidungen angesichts überbordender und redundanter Informationen, kaum umsetzbarer Planbarkeit zeitlicher Erwartungen im Moment zwischen Vergangenheit und Zukunft sowie kaum herstellbare Berechenbarkeit sozialer Erfahrungen (Lang-Wojtasik, 2017, S. 11). Was hier soziologisch beschrieben wird, hat Konsequenzen für die Betrachtung des Zusammenhanges von Gesellschaft und Individuum, ohne direkte Handlungen erwarten zu können. Die angedeuteten Herausforderungen sind das, was gesellschaftlich an Personen adressiert wird. Und Personen adressieren Kommunikationsangebote an die Gesellschaft. Beide Prozesse halten den funktional ausdifferenzierten „Laden“ der Weltgesellschaft am Laufen; allerdings ohne den implizit erhofften Handlungsauftrag und auch ohne direkten Durchgriff zwischen einzelnen Funktionssystemen. Diese agieren nach unterschiedlichen Logiken. Sie können sich gegenseitig irritieren, ohne auf unmittelbare Veränderung hoffen zu können. Das ist folgenreich für übergeordnete Steuerungsfragen. Beispielsweise haben politische Entscheidungen keine direkte Konsequenz für wirtschaftliche Maßnahmen. Vielmehr werden in Gesetze gegossene politische Entscheidungen zu einem neuen kommunikativen Handlungsrahmen, zu der sich ökonomische Logik neu justieren kann und der ggf. juristisch geprüft werden muss. Ethische Optionen können wirtschaftliche Entscheidungen irritieren, jedoch zu keiner direkten Veränderung beitragen.
Der entfesselte globale Markt ist bei alledem keine frei agierende Größe, sondern hat weltweite Verankerungen in nationalen Kontexten, die transnational und interessengeleitet agieren (Beck, 1997). Vor diesem Hintergrund scheinen nationale Staaten und deren Politiken zunehmend als passive $\mathrm{Zu}$ gabe weltgesellschaftlich wahrnehmbarer Entwicklungen (Niederberger \& Schink, 2011, S. 10). Gleichwohl braucht es politisch gesetzte, juristisch wirksame und für andere gesellschaftliche Funktionssysteme leitende Orientierungen, um staatliche Steuerung auf lokaler, regionaler, nationaler und internationaler Ebene annehmen zu können. Dazu helfen ethisch fundierte politische Entscheidungen für das Gemeinwohl genauso, wie ausgleichende volkwirtschaftliche Impulse oder preispolitische Maßnahmen, um den Rahmen abzustecken, in dem ein Handeln von Staatsbürger/-innen ermöglicht wird, das ihren Blick für Solidarität im Sinne eines sozial ausgleichenden Weltbürger/-innentums eröffnet. Innerhalb dieser Rahmenbedingungen kann sich eine Menge ethischer Konsum ereignen, bei dem sich Menschen z. B. bewusst entscheiden, fair gehandelte, saisonal-regionale und ohne Verpackung kaufbare Produkte nachzufragen. Dabei ist im Blick zu behalten, dass dies solidarisch mit dem Blick eines sozialen Ausgleichs geschieht. Bei alledem stellt sich die Schwierigkeit, dass die lokale, regionale, nationale und internationale Ebene unterschiedliche Legitimitätsgrade haben und auf unterschiedliche Weisen miteinander kommunikativ vernetzt sind. Das ist folgenreich, wenn - wie im Falle der Klimakatastrophe - wissenschaftlich erkennbare Notwendigkeiten bekannt sind und in die SDG-Transformationsagenda übersetzt wurden. Um Veränderungen staatlicher Rahmenbedingungen zu erreichen, müssen einerseits verschiedene kommunikative Prozesse durchlaufen werden und andererseits führen kommunikative Adressierungen an Menschen zu keinen direkten Wandlungsprozessen, die über individuelle Aktivitäten hinausgehen: Selbst wenn sich eine weiterführende Schule in einer mittelgroßen europäischen Gemeinde entscheidet, nur noch fair gehandelte und lokal produzierte vegetarische Produkte zu verwenden, die Heizung klimaneutral zu betreiben, den Strom aus erneuerbaren Energien zu beziehen, nur noch auf lokale Unterrichtsmaterialien und dezentrale Verkehrsmittel zu setzen, muss sich im umgebenden Landkreis, Bundesland oder Land gar nichts verändern. Möglicherweise wird diese Schule zu einem Modell für andere Schulen und auch weltweit und ermöglicht Orientierungen für andere Kommunen. Kausal ist dies jedoch nicht. Wenn diese Erkenntnis leitend ist, erscheint die Erprobung neuer Ansätze zur Realisierung der UN-Transformationsagenda als pragmatisches Hoffnungsprogramm (Leal Filho, 2019).

Die große globale Gratwanderung ereignet sich zwischen der Deckung von Bedürfnissen nach Wohlstand einerseits, und dem Sicherstellen einer Zukunft innerhalb der planetarischen Grenzen andererseits. Um hier Wandel zu erhoffen, können Bildungsprozesse initiiert werden, die sich am Weltkollektiv orientieren; also mit der beschriebenen Einheit der Differenz kommunikativer Offerten der Weltgesellschaft und Handlungsoptionen für eine Weltgemeinschaft umgehen können. Das Zusammenleben der globalen Gesellschaft in ihrer Vielfalt und Pluralität ist damit die Voraussetzung für eine nachhaltig überlebensfähige Welt (Fanghanel \& Cousin, 2012) und muss im Zentrum aller Bildungsprozesse stehen. 


\section{These 3: Bildung und Lernen für das Weltkollektiv}

Bildung und Lernen gelten als Schlüssel für zukunftsfähige globale Transformationsprozesse. Kontinuierlicher und nachhaltiger Zugang zu Bildungsangeboten eröffnet neue Räume des Lernens, Denkens und der Wahrnehmung des Weltkollektivs.

Die These mag vor dem Hintergrund der jahrzehntelangen Debatten um Bildung für alle/Education for All zu simpel klingen. Mitnichten! Wenn (Grund-)Bildung im Sinne eines universalen Bildungsverständnisses die „Eintrittskarte ins Leben“ und für lebenslange Bildungsprozesse ist (Delors, 1996), dann müssen Einrichtungen vorhanden sein oder geschaffen werden, die Menschen jedes Alters eine inklusive, gleichberechtigte und hochwertige Bildung gewährleisten und Möglichkeiten lebenslangen Lernens für alle fördern (UN, 2015). Zugespitzt: Wem kontinuierlich jedwede Möglichkeit versagt bleibt, die Welt mit anderen mündig - und damit selbstbefreiend - zu erschließen, dem wird die Teilhabe an der Weltgemeinschaft vorenthalten (Lang-Wojtasik \& Erichsen-Morgenstern, 2019). In diesem Sinne wird dann die besondere Qualität des Untertitels im aktuellen UNESCO Global Monitoring Report deutlich: Alle heißt alle! (UNESCO; 2020). ${ }^{1}$ Im vierten Zielbereich der SDGs ist der gleichberechtigte und diskriminierungsfreie $\mathrm{Zu}$ gang zu und Abschluss von kostenloser sowie hochwertiger Primar-/Sekundarbildung mit gesellschaftlich anschlussfähigen Lernergebnissen die Grundlage weiterer Forderungen. Diese sind eingebettet in die pragmatische Vision eines Lebens über die Lebensspanne sowie des Erwerbs von Kenntnissen und Qualifikationen einer Bildung für nachhaltige Entwicklung, die „nachhaltige Lebensweisen, Menschenrechte, Geschlechtergleichstellung, eine Kultur des Friedens und der Gewaltlosigkeit, Weltbürgerschaft und die Wertschätzung kultureller Vielfalt" umfasst (UN, 2015, S. 17).

Hinter diesen politischen Forderungen stehen schultheoretische Fragen konkreter Rahmenbedingungen qualitativ hochwertiger Bildungseinrichtungen sowie Fragen nach Verständnissen und erhofften Reichweiten von Bildung und Lernen. Die Schule hat sich spätestens seit der europäischen Aufklärung als Leitmedium der Bildungsexpansion bewährt (Lang-Wojtasik, 2008). Sie ermöglicht Bildungs- und Lernprozesse in einem geschützten Rahmen ,unter herabgesetztem Risiko des Scheiterns" (Scheunpflug, 2001, S. 245) mit Impulsen auch für andere Bildungsformate über die Lebensspanne.

Bildung ist in einem allgemeinpädagogischen Sinne jenes Medium, über das eine Transformation von Information in Wissen als möglich angenommen wird. Bildung ist ein Prozess, der eine mündige Beschäftigung mit der Wahrnehmung und Gestaltung von Welt ermöglicht, die Menschen umgibt (Treml, 2000, S. 210ff.). Auf der Ebene des Weltkollektivs wäre dies der stetige Austauschprozess von Informations- und Wissensgesellschaft. Letztere generiert erneut Informationsangebote, die durch Bildung zu anderem Wissen werden können. All dies ist gerahmt durch räumliche Entgrenzung (Globalisierung), zeitliche Beschleunigung (Sozialer Wandel), sachliche Entwertung (Kontingenz) und soziale Befreiung (Individualisierung) (ebd., S. 250ff.).

Lernen kann demgegenüber aus erziehungswissenschaftlicher Perspektive als Steigerung der Eigenkomplexität begriffen werden, um einer zunehmenden Außenkomplexität begegnen zu können. Dabei steht die Veränderung und Neuorganisation neuronaler Verbindungen im Mittelpunkt, über die eine Erweiterung des Verhaltensrepertoires ermöglichbar ist (Treml, 1995).

Wird der Fokus von Lernen im Sinne einer Transformation der Welt als offener Prozess betrachtet, ermöglicht dies einen überblickshaften Aufbau des Weltverständnisses (Smith, 1999-2020). Die Konzentration auf Learnification ist das Resultat aus weitgehend voneinander getrennten Entwicklungen in Bildungstheorie, -praxis und -politik (Biesta, 2015). Allerdings: Lernen ist mehr als ein unspezifischer Zweck von Bildung. Lernen umfasst mindestens drei sich gegenseitig beeinflussende Dimensionen: Inhalt, Zweck und Beziehung für weltzentrierte Bildung. Wenn es um erziehungswissenschaftliche Fragen der Transformation geht, ist eine systematische Betrachtung von Bildung und Lernen unabdingbar. Lernen ist ein individueller Prozess, bei dem Transformation - strukturelle Veränderungen im Gehirn - in einem gegebenen Rahmen passiert, ohne diesen zu verändern. Es ist strukturell mit Bildung gekoppelt, die als „Lernprozesse höherer Ordnung“ bezeichnet werden kann, da der „Modus der Informationsverarbeitung" und damit auch die umgebende Welt des sich selbst verändernden Menschen verändert wird (Koller, 2018).

Lernen für ein zukunftsfähiges Weltkollektiv könnte konsequenterweise auf individuelle Lernprozesse bezogen werden, die sich mit der bewusstseinsbezogenen Verarbeitung von Weltinformation beschäftigen und zu einer Veränderung des Individuums beitragen. Darauf bezogene Bildung wäre konsequenterweise der angenommene Rahmen, in dem Weltveränderung möglich wird. Dabei müsste deutlich geworden sein, dass Bildung und Lernen stets transformativ sind (Scheunpflug, 2019). Begriffe wie "Transformative Bildung“ oder "Transformatives Lernen“ erscheinen konsequenterweise als unterkomplex. Denn es geht ja nicht um ein per se neues Bildungs- oder Lernverständnis, sondern um die alte Hoffnung der Menschheit, dass über Bildung und Lernen zu strukturellen Veränderungen des Weltkollektivs beigetragen werden kann. Den beschriebenen Transformationsprozessen müsste transformativ begegnet werden können. Dabei geht es nicht um bloßen Wandel, sondern um strukturelle Veränderungen, die das Überleben der Menschheit als Weltgemeinschaft wahrscheinlich machen. Dazu sind Initiativen jenseits des aktuell Gegebenen nötig, um Zukunftsfähigkeit im Jetzt zu gestalten (BUND \& Misereor, 1996; Schneidewind, 2018).

Dadurch, dass sich die heutige Welt durch eine volatile, ungewisse, komplexe und ambivalente Eigenschaft (VUKAWelt) (Unkrig, 2020) mit einer beispiellosen Schnelligkeit von Veränderungsprozessen charakterisiert, stehen Bildungsträger vor großen Herausforderungen. Während viele Bildungsentscheidungen nach wie vor auf regionaler und nationaler Ebene getroffen werden, dehnt sich gleichzeitig das komplexe und zunehmend unsichere Geflecht der globalisierten Welt weiter aus, für das nächste Generationen vorbereitet werden sollen. Hier könnten universalisierte Lern- und Bildungsverständnisse (Delors, 1996) zu Orientierungen des Weltkollektivs beitragen, um den Spagat hoffnungsvoller und besorgter Reaktionen auf die sichere Unsicherheit bearbeitbar zu denken (Dill, 2013). 
Noch nie dagewesene globale Krisen sind nicht durch bestehende Strukturen und Ansätze zu bewältigen, sondern müssen so wahrgenommen werden, dass über positive und kreative Haltungen etwas Neues daraus entstehen kann (PirbhaiIllich et al., 2017). In diesem Zusammenhang ermöglichen Bildungskontexte, dominante Bezugsrahmen zu transformieren, um als Weltkollektiv gemeinsam neue Wege für ein $\mathrm{Zu}$ sammenleben zu eröffnen. Bildung hat seit jeher den Auftrag, nach menschlich sinnvoller Entwicklung zu streben, was etwas völlig anderes ist als das Streben nach (Weiter-)Entwicklung. Hilfreich erscheint hier die Fokussierung einer „erwachsenen“ (einanderzugewandten), die eine „kindliche” (egozentrische) Lebensweise überwindbar denkt (Biesta, 2015). Bildung trägt dann dazu bei, neue Wege für ein gerechtes und nachhaltiges Zusammenleben auf einem begrenzten Planeten zu beschreiten. Sie strebt nach Möglichkeiten, das eigene Verlangen zu kontrollieren und alte Bezugsrahmen zu verlassen, um neue Vorstellungen entwickeln zu können. Hier bieten Konzeptionen globaler Bildungsarbeit historisch-systematische Orientierungen.

\section{These 4: Strategische Offerten global orientierter Bildungsarbeit}

Development Education, Globales Lernen und Global Citizenship Education können in Bildungskontexten dazu beitragen, das Bewusstsein der Menschen über Lernen lokal zu verändern und eine Veränderung des Weltkollektivs mit globalem Blick zu erhoffen.

Im pädagogischen Diskurs der Transformation haben die Konzeptionen der Development Education/Entwicklungspädagogik, der Global Education/Globalen Lernens sowie Weltbürgerlichen Erziehung/Global Citizenship Education heute eine unterschiedliche Bedeutung. Das hat mit ihrer Historie und Systematik sowie den zugrundeliegenden konzeptionellen Überlegungen zu tun. Entwicklungspädagogik thematisiert einerseits gesellschaftliche Fragen ökonomischer Unter- und Überentwicklung im Rahmen entwicklungsbezogener Bildungsarbeit für globalen Nord-Süd-Dialog. Andererseits ist die Frage relevant, wie sich Menschen als Individuen durch pädagogische und didaktische Anregungen entwickeln lassen. Insbesondere das erste Verständnis ist in Anlehnung an Development Education auch für den Diskurs des Globalen Lernens bedeutsam. Hier ist es zunächst die komparatistische Würdigung in den 1960er-Jahren, die den Begriff auf eine Beschäftigung mit Bildungsproblemen des Globalen Südens konzentriert. Es geht um die begriffliche Verbindung zu einer Pädagogik der Dritten/Einen Welt, entwicklungspolitischer Bildung sowie konkreter Praxisherausforderungen der Nord-Süd-Solidaritätsarbeit mit bildungsbezogenen Fragen. Der gesellschaftliche Entwicklungsbegriff als Programm von Bildungsanliegen entfaltet seine theoretische Spannung zwischen evolutions- und handlungstheoretischen Zugängen, die für Debatten um Globales Lernen in den 1990er-Jahren hoch relevant sind. Letztlich geht es stets darum, wie Entwicklung zwischen Deskription und Normativität beschrieben werden kann (Treml, 1979; 2017; Datta, 1984).

Diese paradigmatische Unterscheidung ist bis heute auch im Globalen Lernen für eine theoretische Beschäftigung mit Fragen der Globalisierung und ihre metatheoretische Einbettung bedeutsam. Dahinter liegen Fragen, ob und wenn ja wie struktureller Wandel im Sinne globaler Nachhaltigkeit und Gerechtigkeit ermöglicht werden kann, welches Gesellschaftsverständnis unterlegt ist und in welchem Zusammenhang dieses zur Handlungsfähigkeit von Menschen in ihrem direkten Umfeld und bezogen auf den Planeten steht. Es geht darum, wie „Bildung in der Weltgesellschaft“ (Seitz, 2002) beschrieben und umgesetzt werden kann. Die Begriffe Global Education und Globales Lernen zirkulieren seit ungefähr drei Jahrzehnten in deutschsprachigen Diskursen und wurden von der kritischen Beschäftigung mit der Kolonialzeit, einer global ausgleichenden Haltung nach dem Zweiten Weltkrieg sowie zunehmender Internationalisierung geprägt. Wichtige historisch-konzeptionelle Bezugspunkte sind im deutschsprachigen Raum die entwicklungspolitische Bildung und Dritte-Welt-Pädagogik (Scheunpflug \& Seitz, 1995; Scheunpflug, 2017). Globales Lernen ist eine Querschnittskonzeption, die sich im Spannungsfeld menschlicher Lernmöglichkeiten zum Umgang mit Verunsicherung, Variationsvielfalt und Risiko sowie einer thematischen Agenda zur Umsetzung von Nachhaltigkeit und Gerechtigkeit bewegt (Lang-Wojtasik \& Klemm, 2017) und in didaktische Bemühungen übersetzt (Scheunpflug \& Schröck, 2002). Durch den Orientierungsrahmen Globale Entwicklung (KMK, BMZ \& Engagement Global, 2016) ist es bildungspolitisch möglich, Themen des Globalen Lernens in die fach- und domänenspezifische Breite kultuspolitischer Hoheiten im deutschsprachigen Raum zu bringen und in länderbezogenen Lehr- und Bildungsplänen zusammen mit anderen Querschnittsthemen zu verankern. Entwicklungen in anderen europäischen Ländern machen Mut, dass es auch dort zu einer immer stärker wahrnehmbareren Verbreitung im Sinne gesellschaftlicher und bildungsbezogener Aktivitäten kommt (Forghani-Arani, Hartmeyer, O'Loughlin \& Wegimont, 2013; Hartmeyer \& Wegimont, 2016; McAuley, 2018; Trindade Dolejšivá, 2018).

Aus heutiger Sicht ergibt es Sinn, Global Citizenship Education (GCED) als eine Weiterentwicklung von Development Education und Globalem Lernen zu begreifen. GCED hat eine Traditionslinie weltbürgerlicher Erziehung (Seitz, 2017) mit immanenten Orientierungen an einem visionären Weltbürger/-innentum. Dies reicht zurück bis in die römische, griechische und indische Antike (Nussbaum, 2020) und entfaltet hohe Relevanz in den Vorboten und Konkretionen europäischer Aufklärung (Treml, 2011). Die im humanistischen Lebensentwurf veranlagte weltbürgerliche Erziehung wurde mit der Gründung des Nationalstaates zurückgelassen, da eine Bildung zur Identifikation der Bürger/-innen mit dem Staat ins Zentrum rückte (Seitz, 2017). Mit der folgenden Phase der Globalisierung in den 1990er-Jahren wurde der Begriff weltbürgerlicher Erziehung wieder vermehrt aufgegriffen und wird heute auch als Globales Lernen verstanden (ebd.).

Die drei Konzeptionen eint ihre internationale Bedeutung und die Überzeugung, dass über Lernen und Bildung eine veränderte Welt möglich ist, die sich an verschiedenen normativen Leitplanken orientiert. Hier treffen sie sich mit anderen Konzeptionen „epochaltypischer Schlüsselprobleme“ (Klafki, 1985/1996) wie Interkulturelle Pädagogik, Friedenspädagogik, Umweltbildung oder Bildung für nachhaltige Ent- 
wicklung (O'Laughlin \& Wegimont, 2003). Ohne die je besondere Geschichte der einzelnen Konzeptionen in Abrede stellen zu wollen, lassen sie sich als Beiträge zu einem partnerschaftlichen Weltbürger/-innentum mit v. a. vier normativen Leitplanken begreifen, die in verschiedener Intensität zum Tragen kommen: „Nachhaltigkeit als bewahrend-wertschätzende Intra-/Intergenerationalität von Mensch und (Um) Welt, Gewaltfreiheit als rahmender Auftrag einer Kultur des Friedens, Gerechtigkeit als Gleichwürdigkeit im Sinne menschenrechtsbezogener Partizipation und Teilhabe, Partnerschaftlichkeit als kooperativer Umgang mit Anderen und Kultur(en)“ (Lang-Wojtasik, 2019b, S. 30).

GCED wird auf höchster internationaler Ebene gefordert (UNESCO, 2014; 2015). Gleichwohl ist das heutige Verständnis der GCED weit von Einheitlichkeit entfernt und es wird konzeptionell vieles darunter gefasst. Bedeutsame Orientierungspunkte finden sich in diesem Zitat: „Education that is based on postcolonial inquiry, critical thinking, and deep engagement that results in changes in learning, action, and both local and global social conditions. This education does not belong to any one faculty or discipline or to either formal or non-formal education ..." (Jorgenson \& Shultz, 2012, S. 16). GCED erfordert einen Aufbau von Beziehungen und Wissensnetzwerken, die sich mit Differenzen und mit sowohl individuellen als auch sozialen Bedürfnissen der Gesellschaft unter Einbezug lokaler und globaler Blickwinkel befassen. Damit geht sie weit über internationale Bildungsbestreungen hinaus, die Bildung als Vehikel ökonomisch handlungsfähigen Humankapitals begreift. In manchen Ansätzen wird politische Bildungsarbeit als Querschnittsanliegen ergänzt (Wintersteiner et al., 2015). Damit wird die Bedeutung des Citizenship im Weltkollektiv jenseits politikdidaktischer Fokussierungen unterstrichen.

Bei alledem bleibt die pädagogische Hoffnung leitend, dass durch Erziehung, Bildung und Lernen die Welt veränderbar sei. Dabei besteht die Gefahr eines unterkomplexen, kausal-technologischen Verständnisses (Luhmann \& Schorr, 1982), das stets mit der Rationalität des unverfügbaren Menschen (Plessner, 1969/2003) rechnen muss. Damit wird auch die Notwendigkeit einer noch stärkeren theoretischen und empirischen Durchdringung des Diskursfeldes unterstrichen. $\mathrm{Ob}$ und wenn ja wie Wirkungen im beschriebenen Feld erzeugt werden können, bleibt umstritten und braucht noch mehr Klarheit über Entwicklung, Bildung und ihre Zusammenhänge zwischen politischem Hoffen und bildungsbezogenem Können (Bergmüller et al., 2019).

\section{Perspektiven}

Es ist ermutigend zu sehen, wie sich die empirische Forschungslandschaft zu globalen Bildungsfragen ausdifferenziert, wie dies im Rahmen des ANGEL (The Academic Network of Global Education and Learning) oder des DERC (The Development Education and Research Center) deutlich wird. Beeindruckend ist die Vielfalt an Zugängen und Thematiken sowie die stets vorhandene Hoffnung auf Innovation im Sinne gesellschaftlicher und pädagogischer Transformation. Eine wichtige Plattform für den Theorie-Praxis-Dialog ist im deutschsprachigen Raum das EWIK-Portal (www.ewik.de).
Um mit der sicheren Unsicherheit im Weltkollektiv umgehen zu können, braucht es ein Verständnis für die Bedeutung und Reichweite der verwendeten Begriffe in ihren je spezifischen Zusammenhängen. Um den non-kausalen Zusammenhang von Wissen und Handeln bildungsbezogen wertschätzen zu können, hilft zunächst die Unterscheidung kommunikativer Offerten der Weltgesellschaft und handlungsbezogenen Optionen der Weltgemeinschaft. Die untrennbare Einheit der Differenz dieser beiden Weltbeschreibungen lässt sich im Begriff des Weltkollektivs bewahren. Sodann ist es erziehungswissenschaftlich relevant, sich der bildungssoziologischen und -philosophischen Wurzeln der Disziplin rückzuversichern, um sich technologisch fokussierten Kompetenzbegriffen zu entziehen. Development Education, Globales Lernen und Global Citizenship Education interessieren sich für die Menschen als Adressat/-innen und Adressierte von Bildungs- und Lernprozessen!

Mit der erwähnten Maastricht-Erklärung wurde auf europäischer Ebene eine Policy vorgelegt, mit der sich neue strategische Spielräume öffneten, um die Arbeit lange existierenden zivilgesellschaftlichen Engagements und v. a. außerschulischer Bildungsangebote zu bündeln (Wegimont, 2017). Wurde Anfang der 2000er-Jahre noch die globale Dimension hervorgehoben, wird heute immer deutlicher, dass es eigentlich um Bildungsarbeit in glokaler (Robertson, 1995) Spannung geht. Es gibt heute keine bildungsbezogene Entscheidung mehr, die sich jenseits der Interdependenz globaler und lokaler Prozesse ereignet. Kinder, Jugendliche und Erwachsene müssten in den je spezifischen Bildungs- und Lernsettings didaktisch mit der Tatsache konfrontiert werden, dass sich Geschehnisse an voneinander entfernten Orten ereignen und sich trotzdem gegenseitig beeinflussen (Giddens, 1995). Development Education, Globales Lernen und GCED können sehr bedeutsame Bildungsbeiträge für resiliente Demokratie sein. Durch die starke Verankerung in der Tradition europäischer Aufklärung ist gleichwohl stets selbstkritisch die Gefahr einer Re-Hegemonisierung im Blick zu behalten. Immerhin waren aufklärerische Gedanken auch der Ausgangspunkt für Kolonialismus und Imperialismus. Das Festhalten an einem ökonomisch begrenzten Ökonomiebegriff, der sich an Wachstum orientiert und so Nachhaltigkeit produzieren soll, ist dysfunktional im Sinne globalen Ausgleichs. Erhoffte Partnerschaft kann zu einem Bumerang werden, weil die Augenhöhe vergessen wurde oder schlicht strukturell kaum realisierbar ist (Lang-Wojtasik, 2020c/i. E.).

In Anbetracht der vielfältigen Formen, die GCED annehmen kann, geht es vor allem um die Frage, welchen Bezugsrahmens sich das Konzept des Weltbürgertums bedient. Ein verantwortungsvolles Global Citizenship Konzept stellt sicher, dass kollektive Maßnahmen nicht zulasten eines Teils aufgrund der Wohlstandsvermehrung eines anderen Teils der Weltgemeinschaft gehen (Wringe, 1999). Wenn also Bildungsprozesse dahingehend einen Beitrag leisten sollen, dass das Weltkollektiv unter sozial verantwortlichen und nachhaltigen Umständen zukünftig zusammen existieren kann, ist eine kritische Auseinandersetzung mit dem GCED Konzept eine stetige Begleiterin (Andreotti, 2006; Oxley \& Morris, 2013). Diese orientiert sich an einem Rahmen, der aus einer postkolonialen Perspektive existierende Machtverhältnisse lokaler und globaler Systeme infrage stellt (Andreotti, 2011; Abdi, Shultz \& Pillay, 2015). 
Wenn der Anspruch von internationalen Bürger/-innen $\mathrm{zu}$ transnationalen Weltbürger/-innen umgesetzt werden soll, braucht es Klarheit über das unterlegte Bürger/-innen-Verständnis jenseits von Nationen und Staatsbürgerschaften. Status- und Identitätsfragen müssen zwischen Nation, Kontinent (z. B. Europa) und Welt neu justiert werden. Damit sind auch Gefühle, damit assoziierte Zugehörigkeitsfragen in einer Gemeinschaft mit anderen sowie praktische Partizipationsoptionen verbunden (UNESCO, 2015, S. 15; Wintersteiner et al., 2015, S. 22ff.). Pädagogische und didaktische Bemühungen in gesellschaftlichen Querschnittsfeldern sind stets mit der Gefahr eines moralinsauren Zeigefingers konfrontiert. Dieser kann durch die Beschränkung des Beutelsbacher Konsenses Überwältigungsverbot, Kontroversitätsgebot und Schüler/-innenorientierung - entschärft werden (Ahlheim, 2019). Wenn GCED ernsthaft umgesetzt werden soll, braucht es dazu professionelle Kräfte, die als Global Teacher/Facilitator agieren. Diese müssten selbst ihre Position und Haltung zur Welt klären und zum Umgang mit den benannten Querschnittsherausforderungen im Sinne der Nachhaltigkeit, Gerechtigkeit, Partnerschaftlichkeit und Gewaltfreiheit qualifiziert werden (Darji \& Lang-Wojtasik, 2014; Bourn, 2016).

Bildungsinnovationen in Schulen und außerschulischen Bildungseinrichtungen brauchen Klarheit über das unterlegte Entwicklungsverständnis jenseits ökonomischer Reduktionen und über Entwicklung als historischen Begriff (Lang-Wojtasik, 2018). Dazu gehören auch aufrichtige didaktische Rückblicke mit Zukunftsinteresse (Lang-Wojtasik, 2019b; Scheunpflug, 2019). Im Rahmen der Lehrendenbildung haben sich Netzwerkstrukturen v. a. im Bereich der BNE bewährt, die als Türöffner auch für globale Bildungsfragen genutzt werden können (Keil, Kuckuck \& Faßbender, 2019). Um die beschriebenen Anliegen globalnachhaltiger Bildung und Gerechtigkeit noch stärker bildungspolitisch, -forscherisch und -praktisch voranbringen zu können, ist für Development Education, Globales Lernen und Global Citizenship Education noch viel Luft nach oben. Die Rahmenbedingungen sind in Ansätzen auf europäischer, nationaler und teilweise regionaler Ebene erkennbar. Sie brauchen jetzt jenen „Ruck“ für globale Bildungsarbeit, den der ehemalige Bundespräsident Roman Herzog bereits 1976 eingefordert hatte.

\section{Anmerkungen}

$1 \mathrm{Ob}$ jemand sich auf dieses Angebot einlässt, wenn damit erkennbar Entfremdungstendenzen von eigenen Weltbeschreibungen einhergehen, ist eine lange Debatte in befreiungspädagogischen Konzeptionen (Datta \& Lang-Wojtasik, 2002). Sie wird aktuell greifbar in indigenen Widerstandsformen v. a. im globalen Süden, bei denen „moderne Bildung“ als Teil zivilisatorischer Unterdrückung und Domestizierung begriffen wird.

\section{Literatur}

Abdi, A. A., Shultz, L. \& Pillay, T. (Hrsg.) (2015). Decolonizing Global Citizenship Education. Rotterdam/Boston/Taipei:SensePublishers. https://doi.org/10.1007/978 $-94-6300-277-6$

Ahlheim, K. (2019). Beutelsbacher Konsens? Politische Bildung in Zeiten von AFD und Co. Ulm: Klemm + Oelschläger.

Andreotti, V. (2006). Soft versus critical global citizenship education. Policy \& Practice - A Development, 3, 40-51.
Andreotti, V. (2011). Actionable postcolonial theory in education. New York: Palgrave MacMillan. https://doi.org/10.1057/9780230337794

Arnold, M.-T., Carnap, A. \& Bormann, I. (2017). Bestandaufnahme zur Verankerung von Bildung für nachhaltige Entwicklung in Bildungs- und Lehrplänen. Berlin: Freie Universität Berlin.

BMBF (2020). Bildung für nachhaltige Entwicklung. Nationaler Aktionsplan. Zugriff am 22.10.2020 https://www.bne-portal.de/de/die-bildungsbereiche-des-nationalen -aktionsplans-1717.html

Beck, U. (1997). Was ist Globalisierung? Frankfurt a. M.: Suhrkamp.

Bergmüller, C., Causemann, B., Höck, S., Krier, J.-M. \& Quiring, E. (2019). Wirkungsorientierung in der entwicklungspolitischen Inlandsarbeit. Münster u. New York: Waxmann.

Biesta, G. (2015). What is education for? On good education, teacher judgement, and educational professionalism. European Journal of Education, 50(1), 75-87. http s://doi.org/10.1111/ejed.12109

Bourn, D. (2016). Teachers as agents of social change. International Journal of Development Education and Global Learning, 7(3), 63-77. https://doi.org/10.18546/IJD EGL.07.3.05

Bourn D. (Hrsg.) (2020). The Bloomsbury Handbook of Global Education and Learning. London et al.: Bloomsbury.

Bourn, D., Hunt, F. \& Bamber, P. (2017). A review of Education for Sustainable Development and Global Citizenship Education in Teacher Education. Background Paper for the 2017/18 Global Education Monitoring Report. London: UCL Institute of Education.

Brock, A., de Haan, G., Etzkorn, N. \& Singer-Brodowski, M. (2018). Wegmarken zur Transformation: Nationales Monitoring von Bildung für nachhaltige Entwicklung in Deutschland. Opladen: Barbara Budrich. https://doi.org/10.2307/j.ctvddzt7n

BUND \& Misereor (Hrsg.) (1996). Zukunftsfähiges Deutschland. Ein Beitrag zu einer global nachbaltigen Entwicklung. Studie des Wuppertal Instituts für Klima, Umwelt und Energie. Basel, Boston u. Berlin: Birkhäuser. https://doi.org/10.1007/9783-0348-6070-3

Clemens, I., Hornberg, S. \& Rieckmann, M. (Hrsg.) (2019). Bildung und Erziehung im Kontext globaler Transformationen. Opladen, Berlin u. Toronto: Barbara Budrich. https://doi.org/10.3224/84742174

Darji, B. \& Lang-Wojtasik, G. (2014). Preparing globally competent teachers Indo-German Perspectives. International Journal of Global Learning and Development Education, 6(3), 49-62. https://doi.org/10.18546/IJDEGL.06.3.04

Datta, A. (1984). Theorie und Praxis der Entwicklungspädagogik. Lernprozesse und Krisen. Zeitschrift für internationale Bildungsforschung und Entwicklungspädagogik, 7(4), 3-9.

Datta, A. (2013). Armutszeugnis. Warum heute mehr Menschen hungern als vor 20 Jahren. München: dtv.

Datta, A. \& Lang-Wojtasik, G. (Hrsg.) (2002). Bildung zur Eigenständigkeit. Vergessene reformpädagogische Ansätze aus vier Kontinenten. Frankfurt: IKO.

Delors, J. (1996). Learning. The treasure within. Report to UNESCO of the International Commission on Education for the Twenty-first Century. Highlights. Paris: UNESCO. https://doi.org/10.7788/ijbe.1996.24.1.253

Dill, J. S. (2013). The longings and limits of global citzienship education: The modern pedagogy of schooling in a cosmopolitan age. New York: Routledge. https://doi.org/10. 4324/9780203374665

Fanghanel, J. \& Cousin, G. (2012). "Worldly pedagogy": a way of conceptualising teaching towards global citizenship. Teaching in Higher Education, 17(1), 39-50. https://doi.org/10.1080/13562517.2011.590973

Forghani-Arani, N., Hartmeyer, H., O'Loughlin, E. \& Wegimont, L. (Hrsg.) (2013). Global Education in Europe. Policy, Practice and Theoretical Challenges. Münster et al.: Waxmann.

Giddens, A. (1995). Konsequenzen der Moderne. Frankfurt a. M.: Suhrkamp.

Gore, T. (2015). Extreme carbon inequality: Why the Paris climate deal must put the poorest, lowest emitting and most vulnerable people first. Oxfam. Zugriff am 22.10.2020 http://oxf.am/Ze4e

Gore, T. (2020). Confronting carbon inequality: Putting climate justice at the heart of the COVID-19 re-covery. Oxfam. Zugriff am 26.10.2020 https://oxfamilibrary. openrepository.com/bitstream/handle/ 10546/621052/mb-confronting-carbon-inequality-210920-en.pdf.

Grobbauer, H. (2017). Global Citizenship Education. In G. Lang-Wojtasik \& U. Klemm (Hrsg.), Handlexikon Globales Lernen (2. Auflage, S.115-119). Münster u. Ulm: Klemm \& Oelschläger.

Hartmeyer, H. \& Wegimont, L. (Hrsg.) (2016). Global Education in Europe revisited. Strategies and Structures. Policy, Practice and Challenges. Münster u. New York: Waxmann.

IPCC - Intergovernmental Panel on Climate Change (2018). Global Warming of $1.5^{\circ} \mathrm{C}$. An IPCC special report on the impacts of global warming of $1.5^{\circ} \mathrm{C}$ above pre-industrial levels and related global green-house gas emission pathways, in the context 
of strengthening the global response to the threat of climate change, sustainable development, and efforts to eradicate poverty. Zugriff am 20.10.2020 http://ipcc.ch/report/ $\operatorname{sr} 15 /$

Jorgenson, S. \& Shultz, L. (2012). Global Citizenship Education (GCE) in Post-Secondary Institutions: What is protected and what is hidden under the umbrella of GCE? Journal of Global Citizenship \& Equity Education, 2(1), 1-22.

Keil, A., Kuckuck, M. \& Faßbender, M. (Hrsg.) (2019). BNE-Strukturen gemeinsam gestalten. Fachdidaktische Perspektiven und Forschungen zu Bildung für nachhaltige Entwicklung in der Lehrkräftebildung. Münster u. New York: Waxmann. https://doi. org/10.31244/9783830991588

Klafki, W. (1985/1996). Neue Studien zur Bildungstheorie und Didaktik. Zeitgemäße Allgemeinbildung und kritisch-konstruktive Didaktik. (5. Auflage). Weinheim u. Basel: Beltz.

KMK, BMZ \& Engagement Global (Hrsg.) (2016). Orientierungsrahmen für den Lernbereich Globale Entwicklung im Rahmen einer Bildung für nachhaltige Entwicklung. (2. Auflage). Berlin: Cornelsen.

Koller, H.-C. (2018). Bildung anders denken. Einführung in die Theorie transformativer Bildungsprozesse. (2. Auflage). Stuttgart: Kohlhammer.

Lang-Wojtasik, G. (2008). Schule in der Weltgesellschaft. Herausforderungen und Perspektiven einer Schultheorie jenseits der Moderne. Weinheim u. München: Juventa.

Lang-Wojtasik, G. (2014). The World Society and the Human Being. The possibilities and limitations of global Learning in dealing with change. International Journal of Global Learning and Development Education, 6(1), 53-74. https://doi.org/10.1854 6/IJDEGL.06.1.04

Lang-Wojtasik, G. (2017). Globalisierung und Globales Lernen als ein Epizentrum politischer Erwachsenenbildung!? Ulm: Klemm + Oelschläger.

Lang-Wojtasik, G. (2018). Schulen in der Einen Welt - Perspektiven einer global orientierten Schulentwicklungsforschung. In A. Scheunpflug, H. Simojoki \& M. Schreiner (Hrsg.), Evangelische Schulen und religiöse Bildung in der Weltgesellschaft. Die Bamberger Barbara-Schadeberg-Vorlesungen (S. 97-117). Münster et al.: Waxmann.

Lang-Wojtasik, G. (Hrsg.) (2019a). Bildung für eine Welt in Transformation. Global Citizenship Education als Chance für die Weltgesellschaft. Opladen, Berlin u. Toronto: Barbara Budrich. https://doi.org/10.2307/j.ctvpbnpz8

Lang-Wojtasik, G. (2019b). Globales Lernen als transformative Bildung - Überlegungen zu einer Allgemeinen Didaktik mit weltbürgerlichem Anspruch. In G. Lang-Wojtasik \& S. König (Hrsg.), Die Vielfalt methodischer Zugänge in der Unterrichtsforschung. Beispiele aus Fachdidaktik und Allgemeiner Didaktik (S. 23-48). Ulm u. Münster: Klemm + Oelschläger.

Lang-Wojtasik, G. (2020a). Bildung für Nachhaltigkeit und oder nachhaltiges Lernen? Erziehungswissenschaftliche Überlegungen für Global Citizenship Education. In G. Bade, N. Henkel \& B. Reef (Hrsg.), Politische Bildung: vielfältig - kontrovers - global. Festschrift für Bernd Overwien (S. 181-203). Schwalbach/Ts.: Wochenschau.

Lang-Wojtasik, G. (2020b/i. E.). Great Transition and World Collective - Education for transformation within school. In L. Heidrich, Y. Karakașoğlu, P. Mecheril \& S. Shure (Hrsg.), Regimes of Belonging - Schools - Migrations. Wiesbaden: Springer VS

Lang-Wojtasik, G. (2020c/i.E.). Global Citizenship Education als transformativer Friedensauftrag für resiliente Demokratie. In S. Wahl, T. Nauerth \& S. Silber (Hrsg.), Gewaltfreie Zukunft? Gewaltfreiheit konkret! Dokumentation des pax-christiKongresses 2020.

Lang-Wojtasik, G. (2020d/i.E.). Unsichere Sicherheit und Sichere Unsicherheit Gedanken zu einer „neuen Normalität" im Bildungsbereich mit globaler Perspektive. ZEP - Zeitschrift für internationale Bildungsforschung und Entwicklungspädagogik, 43(3).

Lang-Wojtasik, G. \& Erichsen-Morgenstern, R. M. (2019). Transformation als Herausforderung. Globales Lernen als lebenslanger Bildungsauftrag für alle. In W. Leal Filho (Hrsg.), Aktuelle Ansätze zur Umsetzung der UN Nachhaltigkeitsziele (S. 251270). Wiesbaden: Springer. https://doi.org/10.1007/978-3-662-58717-1_14

Lang-Wojtasik, G. \& Klemm, U. (Hrsg.) (2017). Handlexikon Globales Lernen. (2. Auflage). Münster \& Ulm: Klemm + Oelschläger.

Leal Filho, W. (Hrsg.) (2019). Aktuelle Ansätze zur Umsetzung der UN Nachhaltigkeitsziele. Wiesbaden: Springer. https://doi.org/10.1007/978-3-662-58717-1

LfpB - Landeszentrale für politische Bildung Baden-Württemberg (Hrsg.) (2019). SDGs: Globale Ziele, unterschiedliche Perspektiven?! Friedensbildung - Globales Lernen - Bildung für nachhaltige Entwicklung. Fachtagung, 27. - 28. September 2018, Haus auf der Alb, Bad Urach. Bad Urach: LfpB.

Luhmann, N. (1971). Die Weltgesellschaft. Archiv für Rechts-und Sozialphilosophie, 57, 1-35.

Luhmann, N. (1986). Ökologische Kommunikation. Kann die moderne Gesellschaft sich aufökologische Gefährdungen einstellen? Opladen: Westdeutscher Verlag. https:// doi.org/10.1007/978-3-322-94325-5_21
Luhmann, N. \& Schorr, K. E. (1982). Das Technologieproblem der Erziehung und die Pädagogik. In N. Luhmann \& K. E. Schorr (Hrsg.), Zwischen Technologie und Selbstreferenz: Fragen an die Pädagogik (S. 11-40). Frankfurt a. M.: Suhrkamp.

McAuley, J. (Hrsg.) (2018). The State of Global Education in Europe 2018. Dublin: GENE.

Meadows, D., Meadows, D., Zahn, E. \& Milling, P. (1972). Die Grenzen des Wachstums. Bericht des Club of Rome zur Lage der Menschheit. Stuttgart: Deutsche Verlags-Anstalt.

Müller-Christ, G., Giesenbauer, B. \& Tegeler, M. K. (2018). Die Umsetzung der SDGs im deutschen Bildungssystem - Studie im Auftrag des Rats für Nachhaltige Entwicklung der Bundesregierung. ZEP - Zeitschrift für internationale Bildungsfor schung und Entwicklungspädagogik, 41(2), 19-26. https://doi.org/10.31244/zep.20 18.02 .04

Niederberger, A. \& Schink, P. (Hrsg.) (2011). Globalisierung. Ein interdisziplinäres Handbuch. Stuttgart u. Weimar: J. B. Metzler. https://doi.org/10.1007/978-3-476$05327-5$

Nussbaum, M. (2020). Kosmopolitismus. Revision eines Ideals. Darmstadt: WBG.

O'Loughlin, E. and Wegimont, L. (2003). Executive Summary. In E. O'Loughlin \& L. Wegimoint (Hrsg.), Global Education in Europe to 2015. Strategy, policies, and perspectives. Outcomes and Papers of the Europe-wide Global Education Congress Maastricht, The Netherlands, 15.-17. November 2002. Lissabon: North-South-Centre of the Council of Europe.

Overwien, B. (2016). Bildung für nachhaltige Entwicklung in der Schule. In M. K W. Schweer (Hrsg.), Bildung für nachhaltige Entwicklung in pädagogischen Handlungsfeldern. Grundlagen, Verankerung und Methodik in ausgewählten Lehr-Lern-Kontexten (S. 33-47). Frankfurt a. M.: Peter Lang.

Oxley, L. \& Morris, P. (2013). Global Citizenship: A typology for distinguishing its multiple conceptions. British Journal of Educational Studies, 61(3). https://doi.org/ 10.1080/00071005.2013.798393

Pirbhai-Illich, F., Osberg, D., Martin, F., Chave, S., Griffiths, H. \& Sabbah, M. (2017). Re-thinking the Educational Relationship Seminar Research Report. Zugriff am 22.10.2020 https://www.researchgate.net/publication/322146946_Re-thinking the_Educational_Relation-ship_Seminar_Research_Report?channel=doi\&link$\mathrm{Id}=5 \mathrm{a} 47 \mathrm{e} 020458515 \mathrm{f} 6 \mathrm{~b} 0569 \mathrm{~d} 19 \&$ showFulltext=true

Plessner, H. (1924/2003). Grenzen der Gemeinschaft. Eine Kritik des sozialen Radikalismus. In H. Plessner, Macht und menschliche Natur. GS V(S. 7-133). Frankfurt a. M.: Suhrkamp.

Plessner, H. (1969/2003). Homo absconditus. In H. Plessner, Conditio humana. GS VIII (S. 353-366) Frankfurt a. M.: Suhrkamp.

Robertson, R. (1995). Glocalization, Time-Space and Homogeneity-Heterogeneity. In M. Featherstone, S. Lash \& R. Robertson (Hrsg.), Global modernities (S. 25-44). London et al.: Sage. https://doi.org/10.4135/9781446250563.n2

Scheunpflug, A. (2001). Weltbürgerliche Erziehung durch den heimlichen Lehrplan des Schulsystems? In S. Görgens, A. Scheunpflug \& K. Stojanov (Hrsg.), Universalistische Moral und weltbürgerliche Erziehung. Die Herausforderung der Globalisierung im Horizont der modernen Evolutionsforschung (S. 243-254). Frankfurt a. M.: IKO.

Scheunpflug, A. (2017). Globales Lernen - Geschichte. In G. Lang-Wojtasik \& U. Klemm (Hrsg.), Handlexikon Globales Lernen (2. Auflage, S. 141-144). Münster u. Ulm: Klemm \& Oelschläger.

Scheunpflug, A. (2019). Transformatives Globales Lernen - eine Grundlegung in didaktischer Absicht. In G. Lang-Wojtasik (Hrsg.), Bildung für eine Welt in Transformation. Global Citizenship Education als Chance für die Weltgesellschaft (S. 63-74). Opladen, Berlinu. Toronto: Barbara Budrich.https://doi.org/10.2307/j.ctvpbnpz8.7 Scheunpflug, A. \& Schröck, N. (2002). Globales Lernen. Einführung in eine pädagogische Konzeption zur entwicklungsbezogenen Bildung. (2. Auflage) Stuttgart: Brot für die Welt.

Scheunpflug, A. \& Seitz, K. (Hrsg.) (1995). Die Geschichte der entwicklungspolitischen Bildung. Zur pädagogischen Konstruktion der „Dritten Welt“. Frankfurt a. M.: IKO.

Schneidewind, U. (2018). Die Große Transformation. Eine Einführung in die Kunst gesellschaftlichen Wandels. Frankfurt a. M.: Fischer.

Seitz, K. (2002). Bildung in der Weltgesellschaft. Gesellschaftstheoretische Grundlagen Globalen Lernens. Frankfurt a. M.: Brandes \& Apsel.

Seitz, K. (2017). Weltbürgerliche Erziehung. In G. Lang-Wojtasik \& U. Klemm (Hrsg.), Handlexikon Globales Lernen (2. Auflage, S. 395-401). Münster u. Ulm: Klemm \& Oelschläger.

Smith, M.K. (1999-2020). What is learning? A definition and discussion. The Encyclopedia of Pedagogy and Informal Education. Zugriff am 22.10.2020 https://infed. org/mobi/learning-theory-models-product-and-process/

Surian, A. (2018). Mapping Global Education Discourses: A selected literature review. In D. Trindade Dolejšivá (Hrsg.), Innovation, values and policies in Global Education (S. 23-38). Dublin: GENE.

Trindade Dolejšivá, D. (Hrsg.), Innovation, values and policies in Global Education. Dublin: GENE. 
Tönnies, F. (1887/1922). Gemeinschaft und Gesellschaft. Grundbegriffe der Reinen Soziologie. (5. Auflage) Berlin: Carl Curtius.

Treml, A.K. (1979). Was ist Entwicklungspädagogik? ZEP - Zeitschrift für internationale Bildungsforschung und Entwicklungspädagogik, 2(3), 2-4.

Treml, A. K. (1995). Lernen. In H.-H. Krüger \& W. Helsper (Hrsg.), Einführung in Grundbegriffe und Grundfragen der Erziehungswissenschaft (S. 93-102). Opladen: Leske + Budrich. https://doi.org/10.1007/978-3-663-05653-9_8

Treml, A. K. (2000). Allgemeine Pädagogik. Grundlagen, Handlungsfelder und Perspektiven der Erziehung. Stuttgart et al.: Kohlhammer.

Treml, A. K. (2011). Globalisierung als pädagogische Herausforderung. Möglichkeiten und Grenzen einer weltbürgerlichen Erziehung. In W. Sander \& A. Scheunpflug (Hrsg.), Politische Bildung in der Weltgesellschaft (S. 190-203). Bonn: BfPB.

Treml, A.K. (2017). Entwicklungspädagogik. In G. Lang-Wojtasik \& U. Klemm (Hrsg.), Handlexikon Globales Lernen (2. Auflage. S. 63-69). Münster \& Ulm: Klemm + Oelschläger.

UN (2015). Transforming our world: the 2030 Agenda for Sustainable Development. New York, 25.09.2015. Zugriff am 5.02.2019 https://sustainabledevelopment.un. org/post2015/transformingourworld

UNESCO (2015). Global Citizenship Education. Topics and learning objectives. Zugriff am 23.11.2015 http://unesdoc.unesco.org/images/0023/002329/232993e.pdf

UNESCO (2018). Progress on Education for Sustainable Development and Global Citizenship Education. France. Zugriff am 10.09.2019 https://unesdoc.unesco.org/ ark:/48223/pf0000266176

UNESCO (2020). Inklusion und Bildung: Für Alle heißt für Alle. Weltbildungsbericht. Kurzfassung. Bonn: DUK.

Unkrig E.R. (2020). VUKA - Imperativ unserer Welt. In Unkrig, E. R., Mandate der Führung 4.0: Agilität - Resilienz - Vitalität (S. 1-34). Wiesbaden: Springer Gabler. https://doi.org/10.1007/978-3-658-28492-3_1

WBGU - Wissenschaftlicher Beirat der Bundesregierung Globale Umweltveränderungen (2011). Hauptgutachten: Welt im Wandel. Gesellschaftsvertrag für eine Große Transformation. Berlin: WBGU.

Wegimont, K. (2017). Globale Bildung aus europäischer Sicht. In G. Lang-Wojtasik \& U. Klemm (Hrsg.), Handlexikon Globales Lernen (2., überarbeitete und erweiterte Auflage, S. 112-115). Münster u. Ulm: Klemm + Oelschläger.

Wintersteiner, W., Grobbauer, H., Diendorfer, G. \& Reitmair-Juárez, S. (2015). Global Citizenship Education. Politische Bildung für die Weltgesellschaft. (2. Auflage). Wien: ÖUK. Zugriff am 10.09.2019 http://www.demokratiezentrum.org/filead$\mathrm{min} / \mathrm{media} / \mathrm{pdf} /$ Materialien/GlobalCitizenshipEducation_Final.pdf

Wringe, C. (1999). Issues in Citizenship at national, local and global levels. Development Education Journal, 6, 4-6.

\section{Dr. Gregor Lang-Wojtasik}

geb. 1968, Grund- und Hauptschullehrer; Prof. für Pädagogik der Differenz, Studiendekan der Fakultät I, Direktor des Forschungszentrums für Bildungsinnovation und Professionalisierung an der Pädagogischen Hochschule Weingarten; Arbeitsschwerpunkte: International und Interkulturell Vergleichende Erziehungswissenschaft, Grundbildung und Schultheorie, Wertschätzende/Gewaltfreie Kommunikation und Friedenspädagogik, Qualitativ-interpretative Bildungs- und Unterrichtsforschung, Systemtheoretische Erziehungswissenschaft und Philosophische Anthropologie.

\section{Selina Schönborn}

geb. 1990, Absolventin des Masterstudiengangs Educational Leadership an der Tampere University of Applied Sciences (TAMK) Finnland, Dipl. Systematischer Coach \& Beraterin, Akademische Mitarbeiterin an der Pädagogischen Hochschule Weingarten (Koordinatorin des FuN-Kollegs „Heterogenität gestalten - starke Grundschulen entwickeln“), vorher: ProgrammManagerin im internationalen MBA-Programm der Universität St. Gallen, Arbeitsschwerpunkte: Heterogenität und Differenz in Bildungskontexten, Global Citizenship Education, Bildung für nachhaltige Entwicklung, internationale Vernetzung und Kooperation. 